\title{
Unified constitutive modelling for two-phase lamellar titanium alloys at hot forming conditions
}

\author{
Lei Yang ${ }^{1}$, Nan $\mathrm{Li}^{2, *}$, Baoyu Wang ${ }^{1}$, Jianguo Lin ${ }^{1,2}$, Huijun Zhao ${ }^{1}$, and Wenyu Ma ${ }^{1}$ \\ 1 School of Mechanical Engineering, University of Science and Technology Beijing, Beijing 100083, China \\ 2 Department of Mechanical Engineering, Imperial College London, London SW7 2AZ, UK
}

Received 11 June 2016 / Accepted 4 September 2016

\begin{abstract}
In this paper, a set of mechanism based unified viscoplastic constitutive equations have been established for two-phase titanium alloys with initial lamellar microstructure, which models the softening mechanisms of the alloys in hot forming conditions. The dislocation density, rotation and globularization of lamellar $\alpha$-phase and their effects on flow behaviour can also be modelled. The values of material constants in the equation set have been calibrated, according to stress-strain curves and globularization fractions of lamellar $\alpha$-phase obtained from compression tests at a range of temperatures and strain rates, using a genetic algorithm (GA) based optimisation method. Based on the determined constitutive equations, flow stress and globularization evolution of Ti-17 and TA15 alloys at different temperatures and strain rates were predicted. Good agreements between the experimental and computed results were obtained.
\end{abstract}

Key words: Hot forming, Unified viscoplastic constitutive equations, Lamellar microstructure, Softening mechanism, Two-phase titanium alloys, Globularization

\section{Introduction}

Due to a high strength to weight ratio, good thermal stability and welding performance, two-phase titanium alloys have been widely used in the aerospace industry [1-3]. Two-phase titanium alloys can exhibit different microstructures via different heat treatments and forming conditions, such as fully lamellar, bimodal and fully equiaxed. A microstructure consisting of globular $\alpha$-phase in a $\beta$-matrix or transformed $\beta$ (so-called equiaxed microstructure) possesses a good balance of strength and ductility and is thus more desirable for many service applications [4]. Generally, the desired equiaxed microstructure is obtained by breaking down a lamellar microstructure through subtransus processing. In this process, dynamic globularization of $\alpha$-phase plays an important role.

Most previous research has been focused on kinetics of dynamic globularization and the flow behaviour of two-phase titanium alloys with initial lamellar microstructure through compression or tensile tests. Semiatin et al. [5] investigated the dynamic globularization of Ti-6Al-4V during hot working, and analyzed the strains required for its initiation and completion. Shell and Semiatin [6] studied the effect of initial

\footnotetext{
*e-mail: nan.li@imperial.ac.uk
}

microstructures with different $\alpha$ plate thicknesses on dynamic globularization behaviour of Ti-6Al-4V alloy. Miller et al. [7] studied the flow behaviour and its dependence on the $\alpha$-platelet thickness during hot working of Ti-6Al-4V alloy with lamellar microstructure. Park et al. [8] investigated the origins of flow softening of Ti-6Al-4V alloy with initial lamellar microstructure. In their study flow stress softening was less related to dynamic globularization of lamellar $\alpha$ and rotation and particle coarsening of lamellar $\alpha$ were considered as the dominant mechanisms for flow softening. Wu et al. [9] quantitatively studied the dynamic globularization kinetics of TA15 alloy with a lamellar $\alpha$ microstructure during compression in a temperature range of $860-940{ }^{\circ} \mathrm{C}$ and strain rate range of $0.01-10 \mathrm{~s}^{-1}$. They found that the dynamic globularized fraction increases with increasing strain and temperature but with decreasing strain rate. The initiation and completion strains for dynamic globularization of TA15 were predicted using the Avrami-type equation. Wang et al. [10] investigated the flow behaviour and microstructural evolution of Ti-17 alloy with lamellar microstructure during hot deformation in a two-phase $(\alpha+\beta)$ field. In their study, flow softening was attributed to kinking break up and globularization of lamellae due to deformation strain and, to a small extent, deformation heating. Modelling of dynamic globularization of lamellae during the hot working of titanium alloys, to obtain an 
equiaxed microstructure, plays an important role in process optimisation. It has been reported that the globularization is initiated at a critical strain and the globularization fraction increases with strain in a sigmoid way $[9,11]$. The Avramitype equation is often employed to depict the variation of globularization fraction with strain $[9,11,12]$. A backpropagation artificial neural network (ANN) based model was developed by Wang et al. [13] for predicting the dynamic globularization fraction of Ti-17 alloy during hot forming. These approaches can be used to predict the globularization fraction well; however, microstructural evolution was not coupled to deformation kinetics in the models.

The accuracy of numerical simulation largely depends on how accurately the deformation behaviour of a material is represented by the constitutive equations used [14]. There are three main approaches to model the constitutive relations of titanium alloy. They are: empirical, advanced statistical and physically-based internal state variables [15-17]. In the empirical method, an Arrhenius-type model has been widely used to predict the flow stress of titanium alloy at elevated temperatures [18, 19]. Cai et al. [20] carried out an analysis of the constitutive relations of Ti-6Al-4V alloy using a revised Arrhenius-type model. Good agreements between experimental data and predicted data were obtained. Peng et al. [21] conducted a comparative analysis of the Arrhenius-type equations and an artificial neural network (ANN) model to predict the flow stress of an as-cast Ti60 titanium alloy. They found that the ANN model is more efficient in terms of predicting flow stress. However, they are not deformation mechanism-based models. Porntadawit et al. [18] studied the flow behaviour of Ti-6Al-4V using a modified Arrhenius-type equation, a Cingara equation, and a Shafiei-Ebrahimi equation, and the influence of both work hardening and dynamic recrystallization on the hot deformation behaviour of materials were revealed. Zhao et al. [22] investigated flow behaviour and microstructure a Ti600 titanium alloy and established an Arrhenius-type, multiple-linear regression and artificial neural network model to predict the flow stress of the material. Recently, constitutive models coupling microstructural evolution with deformation have received increasing attention. Gao et al. [23] proposed a model comprising a set of unified physically-based equations coupling microstructural evolution for predicting flow stress and globularization evolution during hot working of two phase titanium alloys with initial lamellar microstructure. Babu and Lindgren [24] established a dislocation density based model for plastic deformation and globularization of Ti-6Al-4V. Bai et al. [4] developed mechanism-based unified elastic-viscoplastic constitutive equations coupling globularization of secondary $\alpha$ phase, dislocation density, plastic deformation induced temperature increase and phase transformation, to model flow softening of Ti-6Al-4V. The experimental study indicates that the dominant softening mechanism for two phase titanium alloys with initial lamellar microstructure is slip mode change and the main deformation occurs within the $\beta$ phase [25]. However, there are no unified mechanism-based viscoplastic constitutive equations available to consider the deformation mechanisms and microstructural evolution of two-phase titanium alloys during hot forming. Thus further work is required.
In this paper, a set of mechanism based unified viscoplastic constitutive equations for two phase titanium alloy with initial lamellar microstructure under hot working conditions was developed. It couples the transformation of slip mode due to kink and rotation of lamellar- $\alpha$ phase, as well as the globularization evolution of lamellar- $\alpha$ phase. The unified constitutive model was applied to hot working of Ti-17 and TA15 alloys. In addition, the prediction accuracy of the constitutive descriptions was evaluated through the average absolute relative error (AARE) and correlation coefficient $(R)$. The results show that the proposed model can accurately describe the flow stress globularization relation during hot forming of two phase titanium alloys with an initial lamellar colony microstructure.

\section{Model description}

\subsection{Viscoplastic flow rule}

During hot deformation, an alloy is deformed viscoplastically and a flow rule in terms of power law is used to describe the relation between strain rate and flow stress [26]:

$$
\dot{\varepsilon}_{p}=\left\langle\frac{\sigma-H-k}{K}\right\rangle^{n}
$$

where $\sigma$ is flow stress, $\dot{\varepsilon}_{p}$ is the rate of plastic strain with deformation time (the definite of time is applicable to the rate of other state variables in this work), $k$ is the initial elastic limit which is temperature dependent. $H$ is an internal variable representing the isotropic hardening which expands the yield surface of the material. $K$ is a parameter related to viscous stress, and $n$ is viscous exponent. A higher value of $\mathrm{n}$ indicates a less variation in stress for a certain change of straining rate, that is, the viscoplastic effects are weaker. Both $K$ and $n$ are temperature dependent. The Macaulay brackets $\langle\cdot\rangle$ ensure that, when $(\sigma-H-k)>0$, i.e., when effective stress is outside the elastic domain, equation (1) is tenable.

\subsection{Evolution of dislocation density}

It is difficult to measure the absolute dislocation density of a material experimentally. Therefore, a normalized dislocation density defined by Lin et al. [26, 27] is employed:

$$
\bar{\rho}=1-\frac{\rho_{0}}{\rho}
$$

where $\rho_{0}$ is the initial dislocation density and $\rho$ is the instantaneous dislocation density during deformation. The normalized dislocation density equals zero $(\bar{\rho}=0)$ in the virgin material. At large deformation, $\rho \gg \rho_{0}$, thus $\bar{\rho} \rightarrow 1.0$, which corresponds to the saturated state of a dislocation network of the material.

With regards to high temperature deformation mechanisms, i.e. static and dynamic recoveries and globularization of lamellar $\alpha$, an evolution equation of the normalized dislocation density is proposed as [26]:

$$
\dot{\bar{\rho}}=A_{1}(1-\bar{\rho})\left|\dot{\varepsilon}_{p}\right|-A_{2} \bar{\rho}^{C_{1}}-C_{2} \frac{\bar{\rho}}{(1-S)} \dot{S}
$$



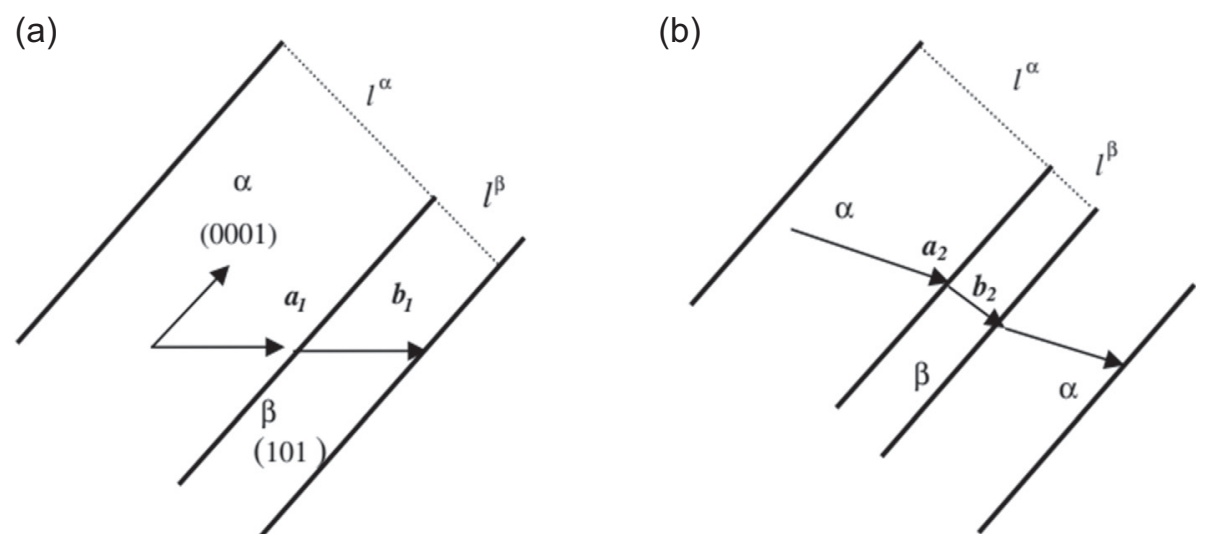

Figure 1. Schematics of (a) soft and (b) hard slip modes in titanium alloys with lamellar microstructure [30] $\left(l^{\alpha}\right.$ and $l^{\beta}$ are the thicknesses of $\alpha$ and $\beta$ lamellae, respectively).

where $A_{1}$ and $A_{2}$, are temperature dependent parameters, $C_{1}$ and $C_{2}$ are material constants, and $S$ is an internal variable representing the globularization of lamellar $\alpha$. The first term represents the accumulation of dislocations due to plastic deformation and dynamic recovery. The second term models the static recovery of dislocations. The third term is related to the reduction of dislocation density due to the globularization of lamellar $\alpha$, which is mainly achieved through recrystallization $[12,27]$. More details of the evolution of globularization, $S$, is given in Section 2.5.

\subsection{Modelling of isotropic hardening}

Isotropic hardening in the studied type of alloys during plastic deformation is expressed as follows:

$$
H=H_{A}+H_{B}
$$

where $H_{A}$ represents isotropic strain hardening and $H_{B}$ represents the grain boundary strengthening at the $\alpha / \beta$ interfaces.

Strain hardening is due to the accumulation of dislocations. A few of the mobile dislocations remain locked in the material lattice, and interact with other dislocations, forming new obstacles to dislocation slip. A proportional relationship existing between the flow stress and the square root of the dislocation density has been confirmed in previous studies $[28,29]$.

$$
H_{A}=B \bar{\rho}^{0.5}
$$

where $B$ is a temperature dependent material parameter, $\bar{\rho}$ is the normalized dislocation density. The internal variable $H_{A}$ presents not only the isotropic hardening caused by stacking and interaction of dislocations, but also allows for material softening resulting from a decrease in the dislocation density. The formulation of $H_{B}$ is detailed in Section 2.4.

\subsection{Modelling of the softening mechanism}

The main softening mechanism of two-phase titanium alloys with colony lamellar microstructure is that the slip mode turns from hard slip mode into soft slip mode [25], which is caused by kink and rotation. The plastic deformation of two-phase titanium alloys with colony microstructure is activated through two types of slip mode, namely the hard and the soft slip mode [30,31], as illustrated in Figures 1a and $1 \mathrm{~b}$, respectively. The soft slip mode corresponds to systems in which dislocations glide parallel to the interface and transmit freely across $\alpha / \beta$ interfaces due to the presence of common slip system between the two phases [25, 30]. The hard slip mode, on the other hand, corresponds to systems in which slip transmission is impeded by $\alpha / \beta$ interfaces due to the absence of a common slip system between them. Therefore, the resistance of the hard slip mode is higher than that of the soft one.

The activation of these two slip modes depends on the misorientation $\theta$ between lamella and stress direction, as shown in Figure 2. When $\theta$ equals $45^{\circ}$, the soft slip mode is activated; when $\theta$ equals $0^{\circ}$ or $90^{\circ}$, the hard slip mode is activated. When $\theta$ falls in the range $0^{\circ}-45^{\circ}$ or $45^{\circ}-90^{\circ}$, the slip mode is somewhere between hard and soft slip mode. With the increase of strain, the slip mode turns into soft slip mode gradually, resulting in flow softening $[25,30,31]$.

Based on the mechanism discussed above, a normalized variable $x_{1}$ is introduced to describe the transition of slip mode in a colony.

$$
x_{1}=1-\frac{\theta}{\theta_{0}}
$$

where $\theta_{0}$ is the misorientation of soft slip mode of a lamella $\left(45^{\circ}\right), \theta$ represents the angle between lamellae and stress orientation $\left(0^{\circ} \leq \theta \leq 45^{\circ}\right)$.

However, it is not practical to experimentally measure the current value of $\theta$ between lamella and stress orientation for each colony in application. In this study, a normalized variable $x$ is adopted to describe the extent of slip mode transition from hard slip mode to soft slip mode in terms of overall response of the material, representing the average behaviours of colonies with different misorientation angles. The transition of slip mode develops with increasing plastic strain and the internal variable $x$ is suggested to be formulated as below [30, 31]:

$$
\dot{x}=C_{3}(1-x)\left|\dot{\varepsilon}_{p}\right|^{\gamma}
$$

where $C_{3}$ and $\gamma$ are material constants. 


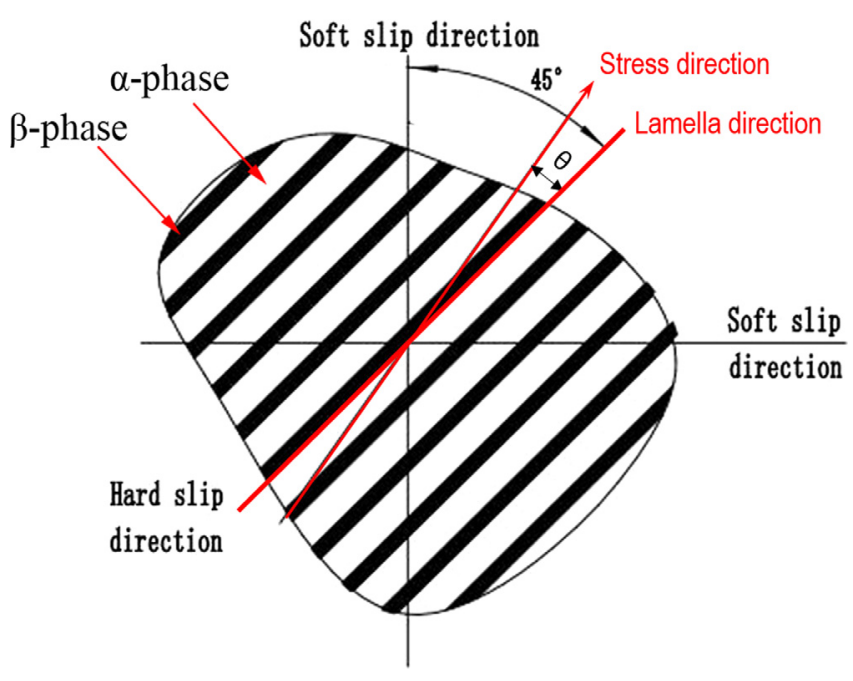

Figure 2. Schematic of the misorientation $(\theta)$ between lamella and principal stress in a colony of Titanium alloys with lamellar microstructure.

Considering the loss of strengthening effect with the rotation of $\alpha$ lamellae, equation (8) is used to describe the phenomena:

$$
H_{B}=(1-x) \Delta k_{c}
$$

where $\Delta k_{c}$ is a temperature and strain rate dependent parameter, representing the interface strength of $\alpha / \beta$, expressed as:

$$
\Delta k_{c}=k_{c 0} \dot{\varepsilon}_{p}^{k_{c 1}} \exp \left(Q_{k c} R_{\mathrm{g}} T\right)
$$

where $k_{c 0}$ and $k_{c 1}$ are constants, $Q_{k c}$ is activation energy, $R_{\mathrm{g}}$ is the gas constant, and $T$ is absolute temperature in $\mathrm{K}$.

\subsection{Modelling of dynamic globularization of lamellar $\alpha$}

The dynamic globularization of lamellar $\alpha$ is the most important microstructural evolution behaviour in the hot working of titanium alloy with initial lamellar colony microstructure. The mechanism of globularization has been extensively studied and it is found that the globularization is mainly achieved through recrystallization $[12,27]$. Referring to the dynamic recrystallization models proposed by Lin et al. [26] and the globularization equation proposed by Gao et al. [23], the evolution of volume fraction of globularization can be formulated as:

$$
\dot{S}=\eta \dot{\varepsilon}_{p}^{\eta_{1}}\left\langle\varepsilon_{p}-\varepsilon_{c}\right\rangle^{\eta_{2}}(1-S)
$$

where $\eta, \eta_{1}, \eta_{2}$ are material constants, $\varepsilon_{c}$ is critical strain for globularization and can be expressed as:

$$
\varepsilon_{c}=\varepsilon_{c 0} \dot{\varepsilon}_{p}^{\eta_{1}} \exp \left(Q_{\varepsilon} R_{\mathrm{g}} T\right)
$$

Where $\varepsilon_{\mathrm{c} 0}$ and $n_{1}$ are material constants, $Q_{\varepsilon}$ is activation energy associated constant.

\subsection{Formulation of the unified viscoplastic constitutive equations}

The unified constitutive equations for a number of metallic materials have been established [4, 32, 33]. The equations enable a wide range of phenomena to be modelled, such as dislocation density [26] and microstructural evolution [34], based on the deformation mechanisms. Considering that the total strain $\varepsilon_{T}$ can be divided into an elastic strain (the elastic strain can be determined by Hooke's law of linear elasticity) and a plastic strain $\varepsilon_{p}$, a set of unified viscoplastic equations for two-phase titanium alloy takes the form of:

$$
\left\{\begin{array}{l}
\dot{\varepsilon_{p}}=\left(\frac{\sigma-H-k}{K}\right)^{n} \\
H=B \bar{\rho}^{0.5}+(1-x) \Delta k_{c} \\
\dot{\bar{\rho}}=A_{1}(1-\bar{\rho})\left|\dot{\varepsilon}_{p}\right|-A_{2} \bar{\rho}^{C_{1}}-C_{2} \frac{\bar{\rho}}{(1-S)} \dot{S} \\
\dot{S}=\eta \dot{\varepsilon}_{p}^{\eta_{1}}\left(\varepsilon_{p}-\varepsilon_{c}\right)^{\eta_{2}}(1-S) \\
\dot{x}=C_{3}(1-x)\left|\dot{\varepsilon}_{p}\right|^{\gamma} \\
\sigma=E\left(\varepsilon_{T}-\varepsilon_{p}\right)
\end{array}\right.
$$

In this set of equations, considering the initial structure change with temperature, which could affect the constitutive behaviour, the temperature dependent parameters $k, K, B, A_{1}$, $A_{2}, \eta, \eta_{1}, \eta_{2}, C_{3}, \gamma$ and $E$ are defined using the classic temperature-compensated parameters in Arrhenius relations, listed in Table 1. Parameters $\Delta k_{c}$ and $\varepsilon_{c}$ are defined by equations (9) and (11), respectively.

For practical applications, e.g. FE simulation of hot forming processes, the tensorial form of the constitutive model should be used. Therefore, the set of constitutive equations (12) is given in the tensorial form:

$$
\left\{\begin{array}{l}
\dot{\varepsilon_{p}}=\left(\frac{\sigma_{e}-H-k}{K}\right)^{n} \\
\dot{\varepsilon}_{i j}^{p}=\frac{3}{2} \frac{X_{i j}}{\sigma_{e}} \dot{\varepsilon_{p}} \\
H=B \bar{\rho}^{0.5}+(1-x) \Delta k_{c} \\
\dot{\bar{\rho}}=A_{1}(1-\bar{\rho})\left|\dot{\varepsilon}_{p}\right|-A_{2} \bar{\rho}^{C_{1}}-C_{2} \frac{\bar{\rho}}{(1-S)} \dot{S} \\
\dot{S}=\eta \dot{\bar{\varepsilon}}_{p}^{\eta_{1}}\left(\varepsilon_{p}-\varepsilon_{c}\right)^{\eta_{2}}(1-S) \\
\dot{x}=C_{3}(1-x)\left|\dot{\varepsilon}_{p}\right|^{\gamma} \\
\sigma_{i j}=D_{i j}\left(\varepsilon_{i j}-\varepsilon_{i j}^{p}\right)
\end{array}\right.
$$


Table 1. List of temperature dependent parameters. $R_{\mathrm{g}}=8.31 \mathrm{~J} \mathrm{~mol}^{-1} \mathrm{~K}^{-1}$ (universal gas constant); $T$ is the absolute temperature in $\mathrm{K}$.

\begin{tabular}{ccc}
\hline$k=k_{0} \exp \left(Q_{k} / R_{\mathrm{g}} T\right)$ & $A_{2}=A_{20} \exp \left(-Q_{A_{2}} R_{\mathrm{g}} T\right)$ & $C_{3}=C_{30} \exp \left(-Q_{C_{3}} R_{\mathrm{g}} T\right)$ \\
\hline$K=K_{0} \exp \left(Q_{K} / R_{\mathrm{g}} T\right)$ & $\eta=\eta_{0} \exp \left(-Q_{\eta} R_{\mathrm{g}} T\right)$ & $\gamma=\gamma_{0} \exp \left(-Q_{\gamma} /\left(R_{\mathrm{g}} T\right)\right.$ \\
$B=B_{0} \exp \left(Q_{B} / R_{\mathrm{g}} T\right)$ & $\eta_{1}=\eta_{10} \exp \left(-Q_{\eta_{1}} R_{\mathrm{g}} T\right)$ & $E=E_{0} \exp \left(Q_{E} /\left(R_{\mathrm{g}} T\right)\right.$ \\
$A_{1}=A_{10} \exp \left(Q_{A_{1}} / R_{\mathrm{g}} T\right)$ & $\eta_{2}=\eta_{20} \exp \left(-Q_{\eta_{2}} R_{\mathrm{g}} T\right)$ & \\
\hline
\end{tabular}

\section{Model application}

\subsection{Determination of model parameters}

The unified constitutive model established in this work is a set of highly non-linear and tightly coupled ordinary differential equations containing a number of constants. It is difficult to determine the values of the constants from experimental data by traditional gradient-based optimisation search methods. Evolutionary programming optimisation techniques, minimizing the residuals between the computed target values and experimental data, have been usually used to determine the model parameters [35]. The details of the optimisation method and the corresponding numerical procedure for this type of problem are described by Li et al. [36], Lin et al. [37, 38], Cao et al. [39, 40] and Zhou et al. [41]. Referring to the objective function developed by Cao and Lin [39], two sub-objective functions were defined as:

$$
\begin{aligned}
& f_{1}(\mathbf{X})=\frac{1}{M} \sum_{j=1}^{M}\left(\frac{1}{N} \sum_{i=1}^{N_{j}}\left[\ln \left(\frac{\sigma_{i j}^{c}}{\sigma_{i j}^{e}}\right)\right]^{2}\right) \\
& f_{2}(\mathbf{X})=\frac{1}{M^{g}} \sum_{j=1}^{M^{g}}\left(\frac{1}{N^{g}} \sum_{i=1}^{N_{j}^{g}}\left[\ln \left(\frac{S_{i j}^{c}}{S_{i j}^{e}}\right)\right]^{2}\right)
\end{aligned}
$$

where $f_{1}(\mathbf{X})$ and $f_{2}(\mathbf{X})$ are objective function describing the error between the computed and experimental values of flow stress and globularization fraction. $\mathbf{X}$ is the array of material constants within the set of equations; $M$ and $M^{\mathrm{g}}$ denote the number of flow stress-strain curves and globularization fraction - strain curves obtained from the experiments; $N_{j}$ and $N_{j}^{\mathrm{g}}$ are the number of data points on the $j$ th curve; the superscript $c$ denotes the values computed from the material model, and the superscript $e$ denotes the corresponding experimental values.

The experimental flow stress and globularization fraction data at various deformation temperatures and strain rates were used to calibrate the material constants in the unified constitutive model. The global objective function was given by:

$$
f(\mathbf{X})=f_{1}(\mathbf{X})+f_{2}(\mathbf{X})
$$

The numerical integration code used to integrate the equations was programmed using the forward Euler method, in which a constant integral step was applied. The GA parameters used were as follows: population size $=200$, number of generations $=5000$, crossover rate $=0.8$ and mutation rate $=0.05$. Through "trial and error" utilising a detailed understanding of the physical basis of each material constant, the best fit values were obtained incrementally.

\subsection{Application to Ti-17 alloy}

Ti-17 (Ti-5Al-2Sn-2Zr-4Mo-4Cr) is a " $\beta$-rich" $\alpha+\beta$ titanium alloy, due to an $8 \%$ content of $\beta$ stabilizer alloying elements such as molybdenum and chromium. It was developed primarily for gas turbine engine components, such as disks for fan and compressor stages [13]. The regular characteristics of material flow behaviour and globularization during hot working of Ti-17 with initial lamellar microstructure was reported by Wang et al. [10, 12, 13]. It has been found that all the flow stress curves exhibit a noticeably flow softening and globularization of lamellar $\alpha$ phase. The flow softening was found to be caused mainly by the kinking, break-up and globularization of lamellae.

The experimental data of flow stress and dynamic globularization fraction at the deformation temperatures of $780{ }^{\circ} \mathrm{C}$, $800{ }^{\circ} \mathrm{C}$ and $820{ }^{\circ} \mathrm{C}$ and the strain rates of $0.001 \mathrm{~s}^{-1}$, $0.01 \mathrm{~s}^{-1}$ and $0.1 \mathrm{~s}^{-1}$ were used in this work. The values of the constants within the equation (13) for Ti-17 alloy are listed in Table 2.

Comparison between the experimental data (symbols) and the computed values (solid lines) is shown in Figures 3 and 4. As can be seen, the fitted model is consistent with the test results, showing good accuracy in the computation of material flow stress and globularization fraction of Ti-17 during hot deformation. Generally, the flow softening trend and globularization kinetic are correctly fitted.

The predictive capability of the constitutive model has been assessed by statistical measures, viz. correlation coefficient $(R)$ and average absolute error (AARE) listed in Figure 5. It can be seen that the average errors are $5.86 \%$ and $17.27 \%$ for stress and globularization fraction respectively. The linear fits of both flow stress and globularization fraction are good, as the $R$ values are 0.9880 and 0.9950 , respectively. This indicates that the errors are small and the model is reliable.

\subsection{Application to TA15 alloy}

TA15 (Ti-6Al-2Zr-1Mo-1V) alloy, a typical near $\alpha$ titanium alloy, has been widely used in manufacturing structural parts in the aerospace industry due to its excellent high-temperature strength, specific strength, thermal stability, weldability, low growth rate of fatigue crack, and strong corrosion resistance [42-45]. Wu et al. [9] studied the regular characteristics of flow softening and globularization during hot working of TA15 alloy with initial lamellar microstructure. The experimental 
Table 2. Determined values of constants for the equation set (13) for Ti-17.

\begin{tabular}{llll}
\hline Constant & Value & Constant & Value \\
\hline$k_{0}(\mathrm{MPa})$ & $1.57980 E-12$ & $\eta_{0}(1 / \mathrm{s})$ & $7.37601 E+01$ \\
$Q_{k}(\mathrm{~J} / \mathrm{mol})$ & $2.61151 E+05$ & $Q_{\eta}(\mathrm{J} / \mathrm{mol})$ & $4.04988 E+04$ \\
$K_{0}(\mathrm{MPa})$ & $2.30800 E+00$ & $\eta_{10}(-)$ & $9.78279 E-01$ \\
$Q_{K}(\mathrm{~J} / \mathrm{mol})$ & $3.63751 E+04$ & $Q_{\eta 1}(\mathrm{~J} / \mathrm{mol})$ & $4.18173 E+02$ \\
$n(-)$ & $3.72515 E+00$ & $\eta_{20}(-)$ & $4.25440 E-02$ \\
$B_{0}(\mathrm{MPa})$ & $3.19712 E+00$ & $Q_{\eta 2}(\mathrm{~J} / \mathrm{mol})$ & $3.20479 E+04$ \\
$Q_{B}(\mathrm{~J} / \mathrm{mol})$ & $2.91070 E+04$ & $\varepsilon_{c 0}(-)$ & $4.71354 E-09$ \\
$k_{c 0}(\mathrm{MPa})$ & $1.58250 E-06$ & $n_{1}(-)$ & $8.40784 E-02$ \\
$k_{c 1}(-)$ & $1.61220 E-01$ & $Q_{\varepsilon}(\mathrm{J} / \mathrm{mol})$ & $1.51256 E+05$ \\
$Q_{k c}(\mathrm{~J} / \mathrm{mol})$ & $1.66462 E+05$ & $C_{30}(1 / \mathrm{s})$ & $1.78760 E+03$ \\
$A_{10}(-)$ & $7.38511 E-05$ & $Q_{c 3}(\mathrm{~J} / \mathrm{mol})$ & $6.36370 E+04$ \\
$Q_{A 1}(\mathrm{~J} / \mathrm{mol})$ & $1.09729 E+05$ & $\gamma_{0}(-)$ & $1.04805 E+03$ \\
$A_{20}(1 / \mathrm{s})$ & $5.04110 E+15$ & $Q_{\gamma}(\mathrm{J} / \mathrm{mol})$ & $6.31590 E+04$ \\
$Q_{A 2}(\mathrm{~J} / \mathrm{mol})$ & $2.98849 E+05$ & $E_{0}(\mathrm{MPa})$ & $4.55222 E+02$ \\
$C_{1}(-)$ & $2.04105 E+00$ & $Q_{E}(\mathrm{~J} / \mathrm{mol})$ & $2.80070 E+04$ \\
$C_{2}(1 / \mathrm{s})$ & $1.00245 E+00$ & & \\
\hline
\end{tabular}

(a)

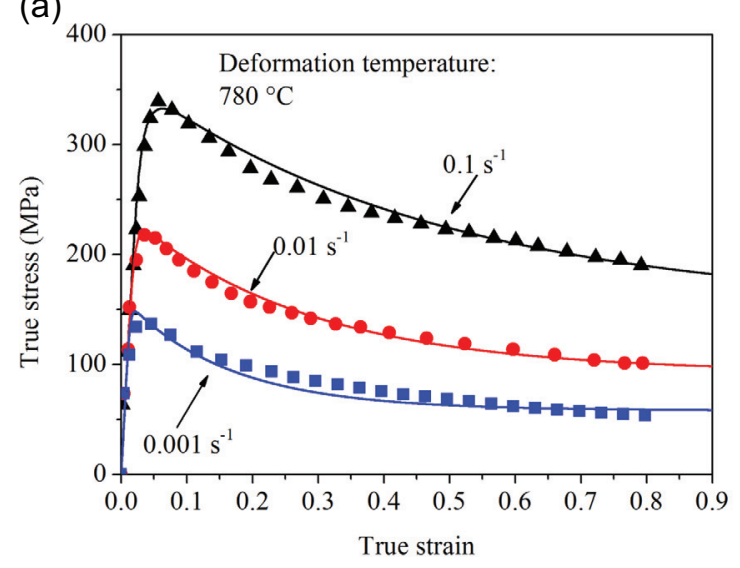

(b)

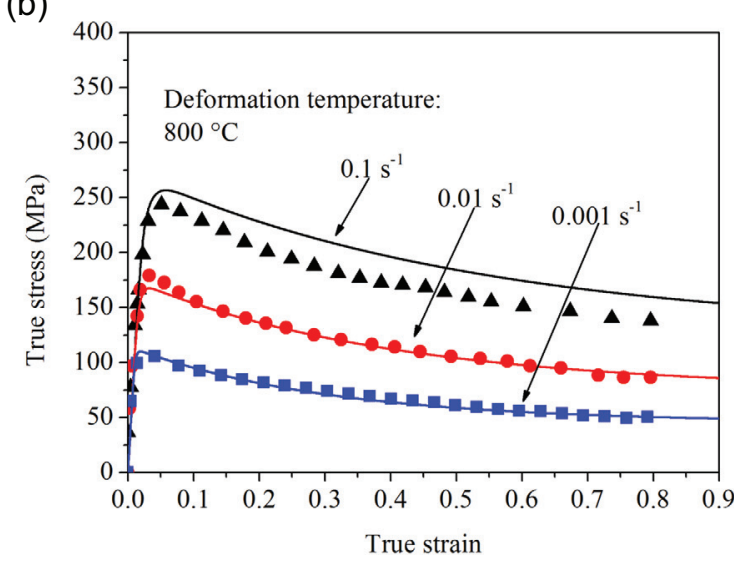

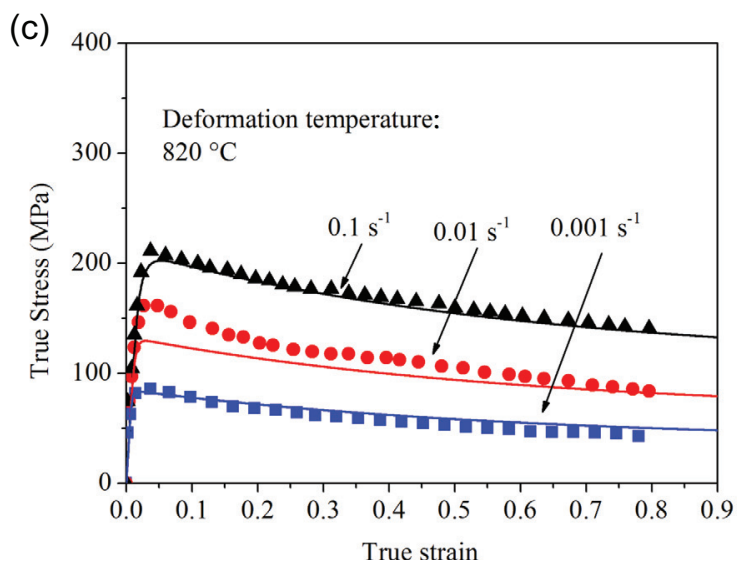

Figure 3. Comparison of the experimental (curves) and computed (symbols) flow stress of Ti-17 with initial lamellar microstructure deformed at the temperatures of (a) $780{ }^{\circ} \mathrm{C}$, (b) $800{ }^{\circ} \mathrm{C}$ and (c) $820{ }^{\circ} \mathrm{C}$ at different strain rates.

flow stress and dynamic globularization fraction in the temperature range of $860{ }^{\circ} \mathrm{C}-940{ }^{\circ} \mathrm{C}$ and the strain rates of 0.1 and $0.01 \mathrm{~s}^{-1}$ were used in this work, as shown in Figures 6 (symbols) and 7 (symbols). Table 3 lists the determined material constants.
Comparison between the experimental data (symbols) and the computed values (solid lines) is shown in Figures 6 and 7, compared with experimental data. As can be seen, from Figures 6 and 7, the fitted curves are in consistent with the test results, showing good accuracy in the computation of material 
(a)

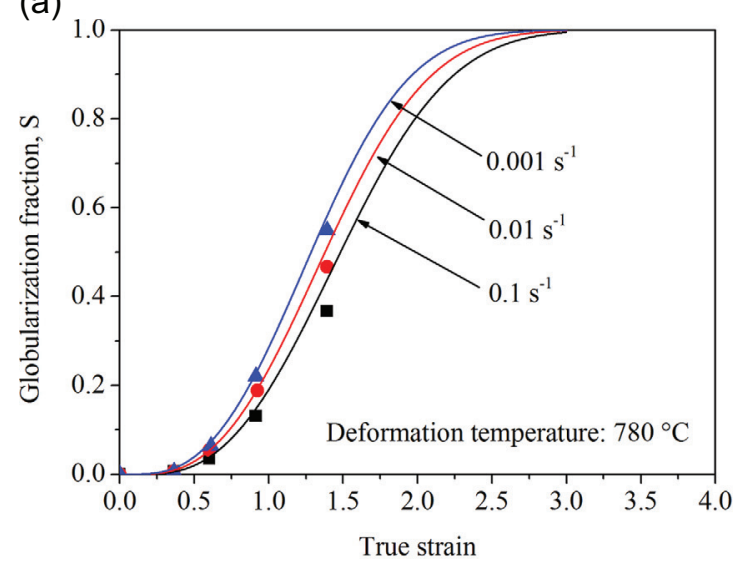

(b)

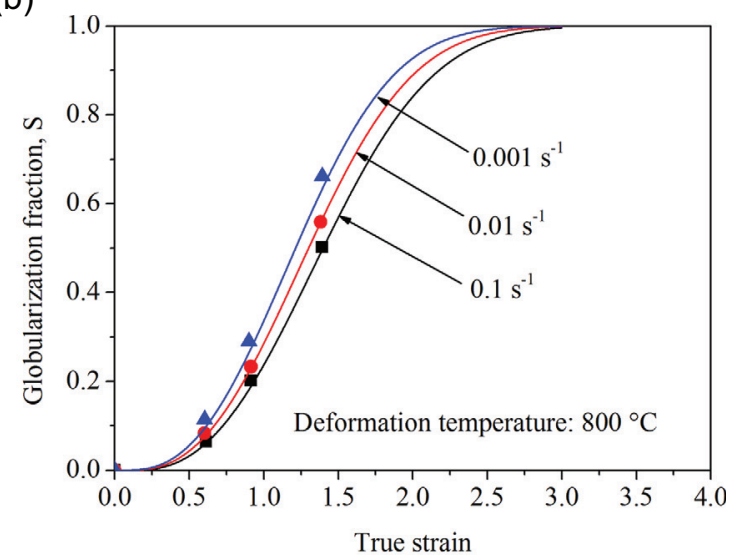

(c)

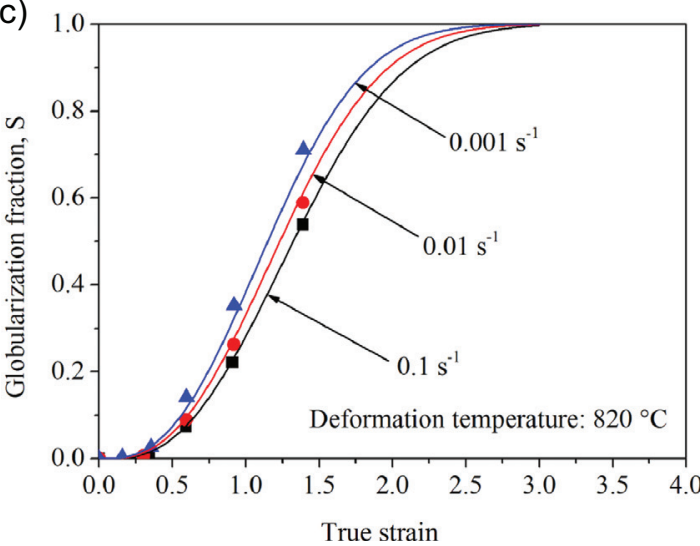

Figure 4. Comparison of the experimental (symbols) and computed (curves) globularization fractions of Ti-17 with initial lamellar microstructure deformed at the temperatures of (a) $780{ }^{\circ} \mathrm{C}$, (b) $800{ }^{\circ} \mathrm{C}$ and (c) $820{ }^{\circ} \mathrm{C}$ at different strain rates.

(a)

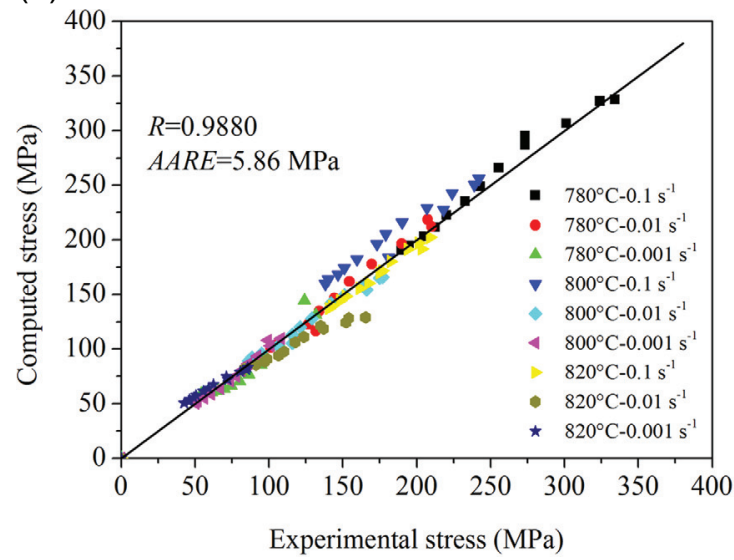

(b)

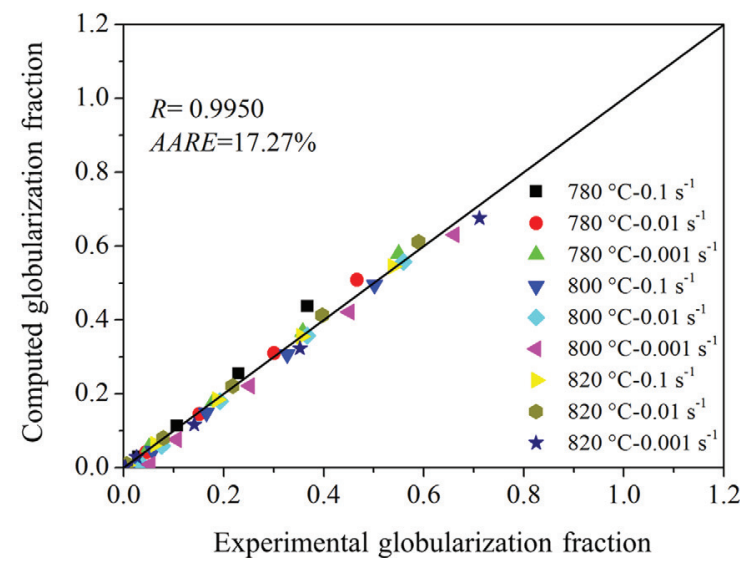

Figure 5. Correlation between the experimental and computed data of Ti-17: (a) flow stress, (b) globularization fraction.

flow stress and globularization fraction of TA15 during hot deformation. Generally, the flow softening trend and globularization kinetic were correctly fitted.

The prediction capability of constitutive model has been assessed by statistical measures, viz. correlation coefficient $(R)$, average absolute error $(A A R E)$, and its standard deviation $(S D)$, listed in Figure 8. It can be seen that the average errors are $4.17 \%$ and $4.49 \%$ for flow stress and globularization fraction, respectively. Considering the correlation coefficient shown in Figure 8, both computed flow stress and globularization fraction are good linear fits, as the $R$ values are 0.9901 and 0.9981 , respectively. This indicates that the errors are small and the model is reliable. 
(a)

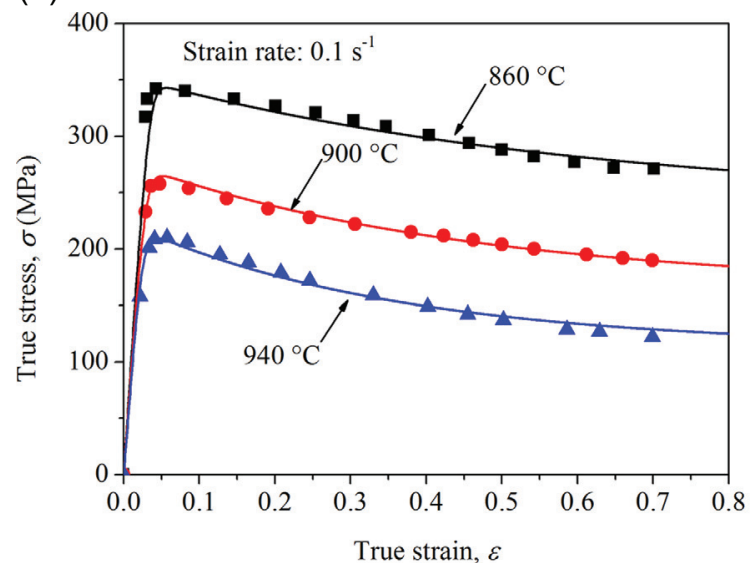

(b)

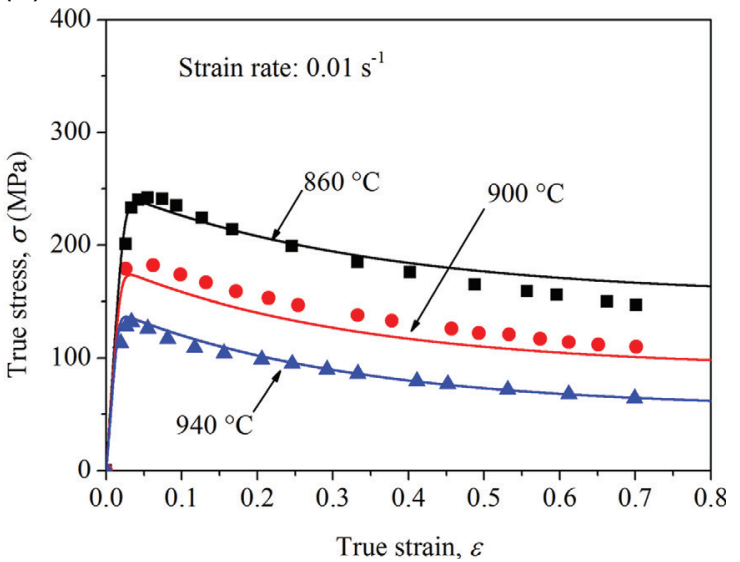

Figure 6. Comparison of the experimental (curves) and computed (symbols) flow stress of TA15 with initial lamellar microstructure deformed at the strain rates of (a) $0.1 \mathrm{~s}^{-1}$ and (b) $0.01 \mathrm{~s}^{-1}$ for different temperatures.

(a)

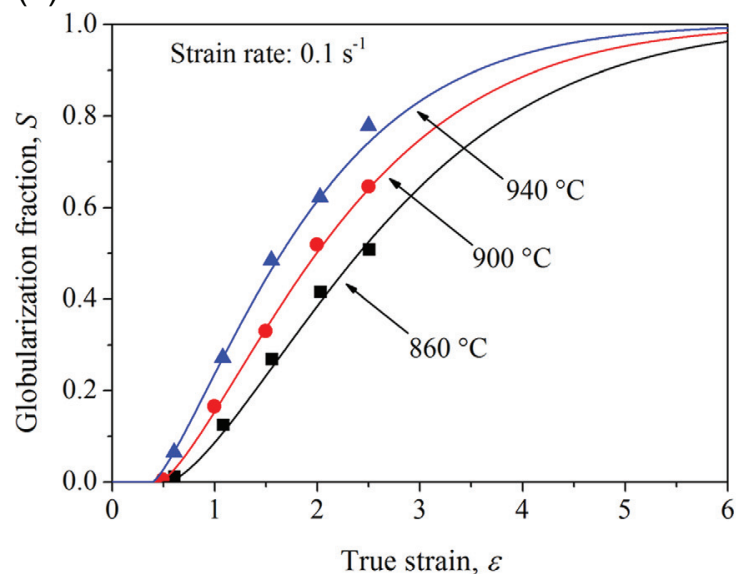

(b)

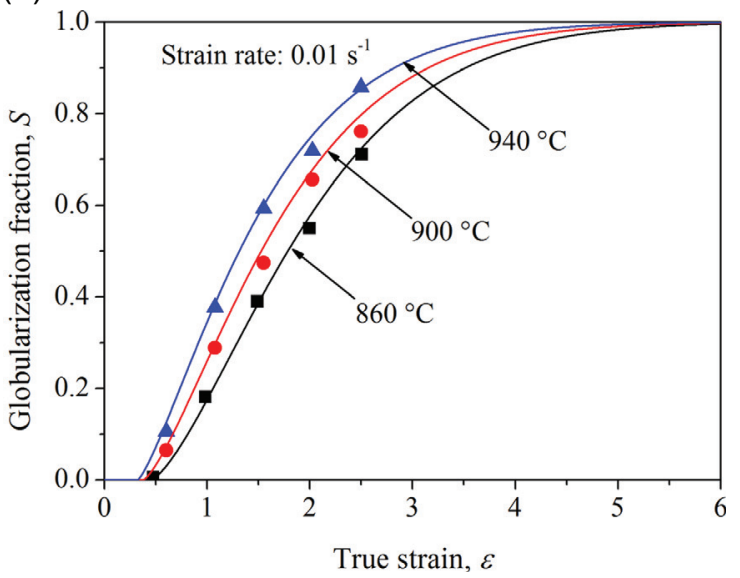

Figure 7. Comparison of the experimental (symbols) and computed (curves) globularization fraction of TA15 with initial lamellar microstructure deformed at the strain rates of (a) $0.1 \mathrm{~s}^{-1}$ and (b) $0.01 \mathrm{~s}^{-1}$ for different temperatures.

Table 3. Determined values of constants for the equation set (13) for TA15.

\begin{tabular}{llll}
\hline Constant & Value & Constant \\
\hline$k_{0}(\mathrm{MPa})$ & $1.1819 E-07$ & $\eta_{0}(1 / \mathrm{s})$ & Value \\
$Q_{k}(\mathrm{~J} / \mathrm{mol})$ & $1.7033 E+05$ & $Q_{\eta}(\mathrm{J} / \mathrm{mol})$ & $6.1049 E+03$ \\
$K_{0}(\mathrm{MPa})$ & $1.0120 E+00$ & $\eta_{10}(-)$ & $9.5064 E+04$ \\
$Q_{K}(\mathrm{~J} / \mathrm{mol})$ & $4.8621 E+04$ & $Q_{\eta 1}(\mathrm{~J} / \mathrm{mol})$ & $3.05559 E+00$ \\
$n(-)$ & $2.2798 E+00$ & $\eta_{20}(-)$ & $1.27087 E+04$ \\
$B_{0}(\mathrm{MPa})$ & $1.1929 E-02$ & $Q_{\eta 2}(\mathrm{~J} / \mathrm{mol})$ & $9.69745 E-04$ \\
$Q_{B}(\mathrm{~J} / \mathrm{mol})$ & $9.0543 E+04$ & $\varepsilon_{c 0}(-)$ & $5.78490 E+04$ \\
$k_{c 0}(\mathrm{MPa})$ & $5.3836 E+01$ & $n_{1}(-)$ & $5.12597 E-03$ \\
$k_{c 1}(-)$ & $8.1979 E-02$ & $Q_{\varepsilon}(\mathrm{J} / \mathrm{mol})$ & $7.93835 E-02$ \\
$Q_{k c}(\mathrm{~J} / \mathrm{mol})$ & $8.3708 E+03$ & $C_{30}(1 / \mathrm{s})$ & $4.50443 E+04$ \\
$A_{10}(-)$ & $2.3894 E-05$ & $Q_{c 3}(\mathrm{~J} / \mathrm{mol})$ & $1.67683 E+06$ \\
$Q_{A 1}(\mathrm{~J} / \mathrm{mol})$ & $1.5625 E+05$ & $\gamma_{0}(-)$ & $1.35601 E+05$ \\
$A_{20}(1 / \mathrm{s})$ & $5.7729 E+08$ & $Q_{\gamma}(\mathrm{J} / \mathrm{mol})$ & $2.18746 E+01$ \\
$Q_{A 2}(\mathrm{~J} / \mathrm{mol})$ & $1.7208 E+05$ & $E_{0}(\mathrm{MPa})$ & $3.16805 E+04$ \\
$C_{1}(-)$ & $1.52537 E+00$ & $Q_{E}(\mathrm{~J} / \mathrm{mol})$ & \\
$C_{2}(1 / \mathrm{s})$ & $1.42757 E+00$ & & $2.70963 E+02$ \\
\hline
\end{tabular}


(a)

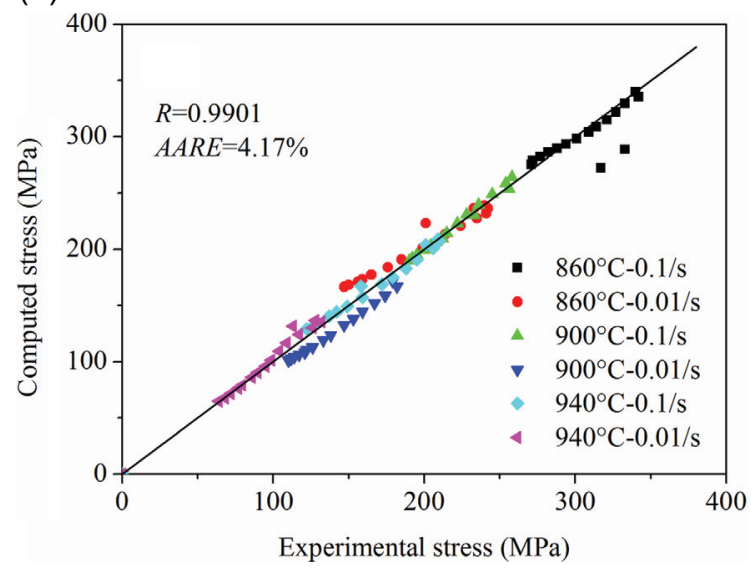

(b)

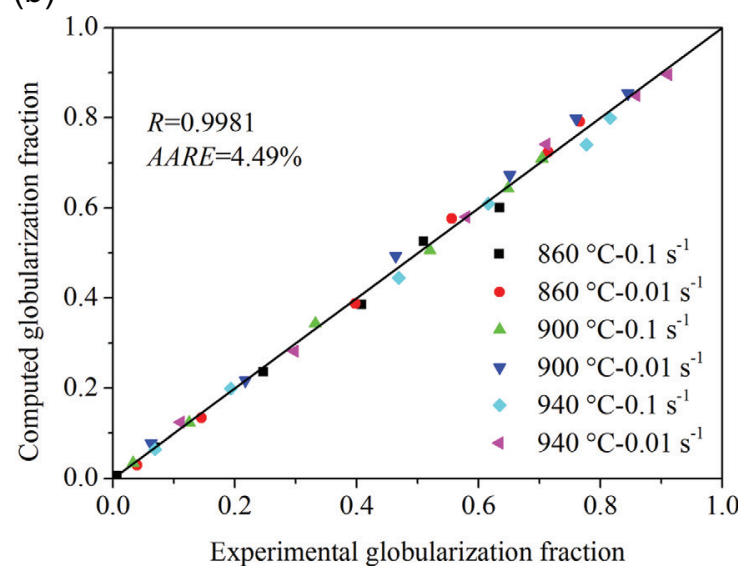

Figure 8. Correlation between the experimental and computed data of TA15: (a) flow stress, (b) globularization fraction.

\section{Conclusions}

Considering the dominant mechanisms of deformation and microstructural evolution, a set of unified viscoplastic constitutive equations has been formulated and determined for the prediction of flow stress and globularization evolution in two-phase titanium alloys with initial lamellar microstructure during hot forming. In this model, the flow stress includes initial yield stress and isotropic hardening. Based on physical mechanisms, the formulated isotropic hardening equation consists of two terms which are strain hardening and boundary strengthening. The globularization evolution of $\alpha$ lamella is modelled as the function of strain and strain rate. The flow softening is modelled by introducing an internal variable $x$ describing the transformation from hard slip mode to soft slip mode and the dislocation density decrease caused by globularization of $\alpha$ lamella. The values of the material constants within the equations are determined using the genetic algorithm based optimisation method. The model has been applied to Ti-17 and TA15 which are both two-phase titanium alloys with initial lamellar microstructures. Statistical analysis indicates that the proposed unified viscoplastic constitutive equations can be used to accurately describe the flow softening and microstructure globularization behaviours of the alloys under hot deformation conditions.

\subsection{Implications and influences}

In this paper, a set of mechanism based unified viscoplastic constitutive equations have been presented for modelling the dominant softening mechanism and the flow stress features. The equation set has been determined from experimental data of Ti-17 and TA15 alloys and close agreements between experimental and computational results have been achieved. These have demonstrated the applicability of the developed material model.

\section{Compliance with Ethical Standards}

The author, Dr. Lei Yang has received the research grant of Doctoral Training Program from Higher Education of China
(No. 20120006110017); Professor Baoyu Wang has received research grant from Beijing Laboratory of Metallic Materials and Processing for Modern Transportation. The other authors declare that they have no conflict of interest.

\section{References}

1. R.R. Boyer, Mater. Sci. Eng. A 213 (1996) 103-114.

2. N.V. Lopatin, Int. J. Mater. Form. 6 (2013) 459-465.

3. C.C. Leyens, Titanium and titanium alloys, Wiley-VCH, Weinheim, 2003.

4. Q. Bai, J. Lin, T.A. Dean, D.S. Balint, T. Gao, Z. Zhang, Mater. Sci. Eng. A 559 (2013) 352-358.

5. S.L. Semiatin, V. Seetharaman, I. Weiss, Mater. Sci. Eng. A 263 (1999) 257-271.

6. E.B. Shell, S.L. Semiatin, Metall. Mater. Trans. A 30 (1999) 3219-3229.

7. R.M. Miller, T.R. Bieler, S.L. Semiatin, Scr. Mater. 40 (1999) 1387-1393.

8. C.H. Park, J.H. Kim, Y. Hyun, J. Yeom, N.S. Reddy, J. Alloys Compd. 582 (2014) 126-129.

9. C. Wu, H. Yang, X. Fan, Z. Sun, Trans. Nonferrous Met. Soc. China 21 (2011) 1963-1969.

10. K.X. Wang, W.D. Zeng, Y.Q. Zhao, Y.J. Lai, X.M. Zhang, Y.G. Zhou, Mater. Sci. Technol. 27 (2011) 21-28.

11. X. Ma, W. Zeng, F. Tian, Y. Zhou, Mater. Sci. Eng. A 548 (2012) 6-11.

12. K. Wang, W. Zeng, Y. Zhao, Y. Lai, Y. Zhou, Mater. Sci. Eng. A 527 (2010) 2559-2566.

13. K. Wang, W. Zeng, Y. Zhao, Y. Shao, Y. Zhou, Mater. Sci. Eng. A 527 (2010) 6193-6199.

14. E.L. Odenberger, M. Schill, M. Oldenburg, Int. J. Mater. Form. 6 (2013) 403-416.

15. Y.C. Lin, X. Chen, Mater. Des. 32 (2011) 1733-1759.

16. W. Hammami, G. Gilles, A.M. Habraken, L. Duchêne, Int. J. Mater. Form. 4 (2011) 205-215.

17. F. Chen, Z.S. Cui, J. Chen, Manufacturing Rev. 1 (2014) 6.

18. J. Porntadawit, V. Uthaisangsuk, P. Choungthong, Mater. Sci. Eng. A 599 (2014) 212-222. 
19. X. Peng, H. Guo, Z. Shi, C. Qin, Z. Zhao, Mater. Des. 50 (2013) 198-206.

20. J. Cai, F. Li, T. Liu, B. Chen, M. He, Mater. Des. 32 (2011) 1144-1151.

21. W. Peng, W. Zeng, Q. Wang, H. Yu, Mater. Des. 51 (2013) 95-104.

22. J. Zhao, H. Ding, W. Zhao, M. Huang, D. Wei, Z. Jiang, Comput. Mater. Sci. 92 (2014) 47-56.

23. P. Gao, H. Yang, X. Fan, S. Zhu, J. Alloys Compd. 600 (2014) 78-83.

24. B. Babu, L. Lindgren, Int. J. Plast. 50 (2013) 94-108.

25. H.W. Song, S.H. Zhang, M. Cheng, Z.X. Li, C.X. Cao, C.L. Bao, Acta Metall. Sin. 47 (2011) 462-468.

26. J. Lin, Y. Liu, D. Farrugia, M. Zhou, Philos. Mag. 85 (2005) 1967-1987.

27. H. Song, S. Zhang, M. Cheng, J. Alloys Compd. 480 (2009) 922-927.

28. J. Lin, T.A. Dean, J. Mater. Process. Technol. 167 (2005) 354-362.

29. M.S. Mohamed, A.D. Foster, J. Lin, D.S. Balint, T.A. Dean, Int. J. Mach. Tools Manuf. 53 (2012) 27-38.

30. G. Venkataramani, K. Kirane, S. Ghosh, Int. J. Plast. 24 (2008) 428-454.

31. S.L. Semiatin, Metall. Mater. Trans. A 32 (2001) 1787-1799.
32. Y. Liu, J. Lin, T.A. Dean, D.C.J. Farrugia, J. Strain Anal. Eng. Des. 40 (2005) 571-586.

33. C.L. Lam, Z.S. Shi, X. Huang, Y.L. Yang, Y.S. Zeng, J.L. Lin, Manufacturing Rev. 2 (2015) 19.

34. B.H. Cheong, J. Lin, A.A. Ball, J. Mater. Process. Technol. 134 (2003) 10-18.

35. M. Farahmand-Mehr, P. Fattahi, M. Kazemi, H. Zarei, A. Piri, Manufacturing Rev. 1 (2014) 21.

36. B. Li, J. Lin, X. Yao, Int. J. Mech. Sci. 44 (2002) 987-1002.

37. J. Lin, J. Yang, Int. J. Plast. 15 (1999) 1181-1196.

38. J. Lin, B.H. Cheong, X. Yao, J. Mater. Process. Technol. 125 (2002) 199-205.

39. J. Cao, J. Lin, Int. J. Mech. Sci. 50 (2008) 193-204.

40. J. Cao, J. Lin, T.A. Dean, Int. J. Numer. Methods Eng. 73 (2008) 1094-1112.

41. J. Zhou, B. Wang, M. Huang, Mater. Des. 63 (2014) 738-748.

42. D. He, J. Zhu, S. Zaefferer, D. Raabe, Mater. Des. 56 (2014) 937-942.

43. G. Quan, D. Wu, G. Luo, Y. Xia, J. Zhou, Q. Liu, L. Gao, Mater. Sci. Eng. A 589 (2014) 23-33.

44. X.F. Wang, D.X. Liu, W.D. Li, L. Xu, Y.G. Dong, Manufacturing Rev. 1 (2014) 25.

45. L.H. Lang, K.N. Liu, G.S. Cai, X.Y. Yang, C. Guo, G.L. Bu, Manufacturing Rev. 1 (2014) 9.

Cite this article as: Yang L, Li N, Wang B, Lin J, Zhao H \& Ma W: Unified constitutive modelling for two-phase lamellar titanium alloys at hot forming conditions. Manufacturing Rev. 2016, 3, 14. 